\title{
SECOND ORDER LINEAR OSCILLATION WITH INTEGRABLE COEFFICIENTS ${ }^{1}$
}

\author{
BY JAMES S. W. WONG ${ }^{2}$
}

\section{Communicated by W. Wasow, April 4, 1968}

We are here concerned with the oscillatory behaviour of solutions of the following second order ordinary differential equation:

$$
x^{\prime \prime}+a(t) x=0, \quad\left({ }^{\prime}=d / d t, t \geqq 0\right),
$$

where $a(t)$ is locally integrable in $t$. We call equation (1) oscillatory if all solutions of (1) have arbitrarily large zeros, otherwise, we say equation (1) is nonoscillatory. As a consequence of Sturm's Separation Theorem [1], if one of the solutions of (1) is oscillatory, then all of them are. The same is true for nonoscillatory solutions of (1).

Our primary interest here is to consider equations with integrable coefficients, namely those functions $a(t)$ satisfying

$$
\lim _{T \rightarrow \infty} \int_{0}^{T} a(t) d t<\infty .
$$

In this case, one may introduce the function $A(t)$ defined by

$$
A(t)=\int_{t}^{\infty} a(s) d s, \quad t \geqq 0 .
$$

In contrast to most of the earlier works on this subject, the coefficient $a(t)$ and its iterated integral $A(t)$ are not assumed to be nonnegative here. We shall be interested in those cases when the iterated integral $A(t)$ is again integrable as a function of $t$. A typical example of such is the function $a(t)=\sin \beta t / t, \beta \neq 0$. As far as we know, the range of $\beta$ for which equation (1) is oscillatory or nonoscillatory has not been completely determined. There are two exceptions which offer partial solutions. Using Hartman [1, p. 369, Exercise 7.9], one can deduce that (1) is nonoscillatory if $|\beta|>4$. Willett [3] recently gives results which will yield oscillation of (1) if $|\beta|<2^{1 / 2}$ and nonoscillation if $|\beta|>2^{1 / 2}$. It will be clear from the results below that (1) is also nonoscillatory when $|\beta|=2^{1 / 2}$. We shall discuss further perturbations of this boundary case in an example below.

\footnotetext{
1 Sponsored by the Mathematics Research Center, United States Army, Madison, Wisconsin, under Contract No. DA-31-124-ARO-D-462.

2 Present address: Department of Mathematics, Carnegie-Mellon University, Pittsburgh, Pennsylvania 15213.
} 
The basis for our results is the introduction of a new Riccati integral equation

$$
u(t)=\bar{A}(t)+\int_{t}^{\infty} Q_{A}(s, t) u^{2}(s) d s
$$

where

$$
Q_{A}(s, t)=\exp \left(\int_{t}^{s} 2 A(\tau) d \tau\right), \text { and } \bar{A}(t)=\int_{t}^{\infty} Q_{A}(s, t) A^{2}(\tau) d \tau .
$$

Our main result is the fact that the existence of a solution to equation (4) is equivalent to the nonoscillation of (1). If the function $A(t)$ is again integrable in the sense of (2), then a similar equation may be generated from (4), the existence of solution of which is again equivalent to the nonoscillation of (1). Using these equations, we can derive new oscillation and nonoscillation criteria which are particularly effective with oscillating coefficient $a(t)$ like the example above. We list below a sampling of our results whose proofs together with other details will appear elsewhere.

THEOREM 1. Equation (1) is nonoscillatory if and only if equation (4) has a solution for sufficiently large $t$.

THEOREM 2. If $A(t)$ is integrable, then equation (1) is nonoscillatory if and only if there exists a solution to the equation

$$
w(t)=\overline{\bar{A}}(t)+\int_{t}^{\infty} Q_{\bar{A}}(s, t) w^{2}(s) d s
$$

for sufficiently large $t$, where $Q_{\bar{A}}$ and $\overline{\bar{A}}$ are defined as $Q_{A}$ and $\bar{A}$ with $\bar{A}$ replacing $A$.

THEOREM 3. If there exists a function $B(t)$ such that

$$
\int_{t}^{\infty}(A(s)+B(s))^{2} Q_{A}(s, t) d s \leqq B(t),
$$

then (1) is nonoscillatory.

THEOREM 4. If there exists a nonzero function $C(t)$ such that

$$
\int_{t}^{\infty} \bar{A}^{2}(s) Q_{A}(s, t) d s \geqq \frac{1}{4} \bar{A}(t)+C(t),
$$

and 


$$
\int_{t}^{\infty} C(s) \bar{A}(s) Q_{A}(s, t) d s \geqq \delta C(t),
$$

for some $\delta \geqq \frac{1}{4}$, then (1) is oscillatory.

Theorems 3 and 4 above are motivated by results of Opial [2] and their recent generalizations by Willett [3]. In particular, by taking $B(t) \equiv \bar{A}(t)$ in (6) and $C(t) \equiv \epsilon \bar{A}(t), \epsilon>0$, in (7) and (8), we obtain the corresponding results in [3]. Using equation (5) instead of (4), we obtain oscillation and nonoscillation conditions similar to (6), (7) and (8), with $\bar{A}(t), \bar{A}(t)$ replacing $\bar{A}(t)$ and $A(t)$. Applying Theorems 3 and 4 to the example $a(t)=\alpha \sin \beta t / t^{\gamma}, \gamma>0, \alpha, \beta \neq 0$, we obtain

(i)

$$
\begin{aligned}
& \begin{array}{l}
\gamma>1, \quad \alpha, \beta \text { arbitrary } \\
\gamma=1, \quad|\alpha / \beta| \leqq 1 / 2^{1 / 2}
\end{array} \Rightarrow(1) \quad \text { nonoscillatory. } \\
& \text { (ii) } \begin{array}{l}
\gamma<1, \quad \alpha, \beta \text { arbitrary } \\
\gamma=1, \quad|\alpha / \beta|>1 / 2^{1 / 2}
\end{array} \Rightarrow(1) \quad \text { oscillatory. }
\end{aligned}
$$

In fact, conclusions (i), (ii) remain valid if $A(t)=(\alpha / \beta)\left(\cos \beta t / t^{\gamma}\right)$ $+O\left(1 / t^{\gamma+\epsilon}\right)$ for some $\epsilon>0$. Consider the boundary case: $\gamma=1,|\alpha / \beta|$ $=1 / 2^{1 / 2}$, and the example $a(t)=\alpha \sin \beta t / t+\xi \sin \eta t / t \log t$. Using the above results, we can show that

(i) if $\beta \neq \eta$, then (1) is oscillatory.

(ii) if $\beta=\eta$, then $\alpha \xi>0 \Rightarrow$ oscillation,

$$
\alpha \xi \leqq 0 \Rightarrow \text { nonoscillation. }
$$

\section{REFERENCES}

1. P. Hartman, Ordinary differential equations, Wiley, New York, 1964.

2. Z. Opial, Sur les integrales oscillantes de l'equation differentielle $u^{\prime \prime}+f(t) u=0$, Ann. Polon. Math. 4 (1958), 308-313.

3. D. Willett, On the oscillatory behaviour of the solutions of second order linear differential equations, Ann. Polon. Math., (to appear).

Mathematics Research Center, University of Wisconsin 